\title{
Reconstruction of Patient-Specific 3D Bone Surface from 2D Calibrated Fluoroscopic Images and Point Distribution Model
}

\author{
Guoyan Zheng ${ }^{1}$, Miguel Á.G. Ballester ${ }^{1}$, Martin Styner ${ }^{2}$, and Lutz-Peter Nolte ${ }^{1}$ \\ ${ }^{1}$ MEM Research Center, University of Bern, CH-3014, Bern, Switzerland \\ Guoyan.Zheng@MEMcenter . unibe.ch \\ ${ }^{2}$ Departments of Computer Science and Psychiatry, University of North Carolina at \\ Chapel Hill, Chapel Hill, NC 27599-3175, USA
}

\begin{abstract}
Reconstruction of patient-specific 3D bone surface from 2D calibrated fluoroscopic images and a point distribution model is discussed. We present a 2D/3D reconstruction scheme combining statistical extrapolation and regularized shape deformation with an iterative imageto-model correspondence establishing algorithm, and show its application to reconstruct the surface of proximal femur. The image-to-model correspondence is established using a non-rigid $2 \mathrm{D}$ point matching process, which iteratively uses a symmetric injective nearest-neighbor mapping operator and 2D thin-plate splines based deformation to find a fraction of best matched 2D point pairs between features detected from the fluoroscopic images and those extracted from the 3D model. The obtained $2 \mathrm{D}$ point pairs are then used to set up a set of $3 \mathrm{D}$ point pairs such that we turn a $2 \mathrm{D} / 3 \mathrm{D}$ reconstruction problem to a $3 \mathrm{D} / 3 \mathrm{D}$ one. We designed and conducted experiments on 11 cadaveric femurs to validate the present reconstruction scheme. An average mean reconstruction error of $1.2 \mathrm{~mm}$ was found when two fluoroscopic images were used for each bone. It decreased to $1.0 \mathrm{~mm}$ when three fluoroscopic images were used.
\end{abstract}

Keywords: point distribution model, surface reconstruction, 2D/3D correspondence, extrapolation, deformation, thin-plate splines.

\section{Introduction}

Constructing three-dimensional (3D) bone surface from a few two-dimensional (2D) calibrated fluoroscopic images is a challenging task. A priori information is often required to handle this otherwise ill-posed problem. In Fleute and Lavallée [1, a point distribution model (PDM) of distal femur was iteratively fitted to the bone contours segmented on the $\mathrm{X}$-ray views by sequentially optimizing the rigid and non-rigid parameters. It utilizes the principle of the shortest distance between the projection ray of an image edge pixel and a line segment on the apparent contour to set up image-to-model correspondence. However, this method requires an explicit contour extraction, which may be quite difficult when the shapes involved become complex or when the background of the images becomes complex. Furthermore, least-squares based fit may result in unstable solution. 
In Benameur et al. [2] 3], a PDM of scoliotic vertebrae was fitted to two conventional radiographic views by simultaneously optimizing both shape and pose parameters. The optimal estimation was obtained by iteratively minimizing a combined energy function, which is the sum of a likelihood energy term measured from an edge potential field on the images and a prior energy term measured from the statistical shape models. No explicit image-to-model correspondence was used. To avoid trapping in a local minimal, their method requires a close initialization.

This paper presents a $2 \mathrm{D} / 3 \mathrm{D}$ reconstruction scheme combining statistical extrapolation and regularized shape deformation with an iterative image-to-model correspondence establishing algorithm, and shows its application to reconstruct the surface of proximal femur. The image-to-model correspondence is established using a non-rigid 2D point matching process, which iteratively uses a symmetric injective nearest-neighbor mapping operator and 2D thin-plate spline (TPS) based deformation to find a fraction of best matched 2D point pairs between features detected from the fluoroscopic images and those extracted from the 3D model. The obtained 2D point pairs are then used to set up a set of $3 \mathrm{D}$ point pairs such that we turn a $2 \mathrm{D} / 3 \mathrm{D}$ reconstruction problem to a $3 \mathrm{D} / 3 \mathrm{D}$ one. No explicit contour extraction from fluoroscopic images is required in the present scheme.

This paper is organized as follows. Section 2 presents the point distribution model. Section 3 describes the iterative image-to-model correspondence establishing algorithm. Section 4 briefly recalls the 3D/3D reconstruction framework introduced in [4]. Section 5 presents the experimental results, followed by the conclusions in Section 6.

\section{Point Distribution Model}

The PDM used in this paper was constructed from a training database consisted of 30 proximal femoral surfaces from above the less trochanter. To optimally align these training surfaces, a sequence of correspondence establishing methods presented in [5] was employed. It started with a SPHARM-based parametric surface description [6] and then was optimized using Minimum Description Length (MDL) based principle as proposed by Davis et al [7.

Following the alignment, the PDM is constructed as follows. Let $\mathrm{x}_{i}, i=$ $0,1, \ldots, m-1$, be $m$ (here $m=30$ ) members of the aligned training surfaces. Each member is described by a vectors $\mathrm{x}_{i}$ with $N$ vertices:

$$
\mathrm{x}_{i}=\left\{x_{0}, y_{0}, z_{0}, x_{1}, y_{1}, z_{1}, \ldots, x_{N-1}, y_{N-1}, z_{N-1}\right\}
$$

The PDM is obtained by applying principal component analysis.

$$
\begin{aligned}
& D=\left((m-1)^{-1}\right) \cdot \sum_{i=0}^{m-1}\left(\mathrm{x}_{i}-\overline{\mathrm{x}}\right)\left(\mathrm{x}_{i}-\overline{\mathrm{x}}\right)^{T} \\
& P=\left(\mathbf{p}_{0}, \mathbf{p}_{1}, \ldots\right) ; D \cdot \mathbf{p}_{i}=\sigma_{i}^{2} \cdot \mathbf{p}_{i}
\end{aligned}
$$

where $\overline{\mathrm{x}}$ and $D$ are the mean vector and the covariance matrix, respectively. 
Then, any one of the instance in this space can be expressed as:

$$
\mathrm{x}=\overline{\mathrm{x}}+\sum_{i=0}^{m-2} \alpha_{i} \mathbf{p}_{i}
$$

And the estimated normal distribution of the coefficients $\alpha_{i}$ is:

$$
p\left(\alpha_{0}, \alpha_{1}, \ldots, \alpha_{m-2}\right)=(2 \pi)^{-\frac{m-1}{2}} \cdot \exp \left(-\frac{1}{2} \sum_{i=0}^{m-2}\left(\alpha_{i}^{2} / \sigma_{i}^{2}\right)\right)
$$

where $\sum_{i=0}^{m-2}\left(\alpha_{i}^{2} / \sigma_{i}^{2}\right)$ is the Mahalanobis distance defined on the distribution.

\section{Image-to-Model Correspondence}

Given a few fluoroscopic images, our task is to establish correspondence between the input images and a model estimated from the PDM. Here we assume that the input images are calibrated and registered to a common coordinate system. And for a pixel in an input image we can always find a projection ray emitting from the focal point of the associated image through the pixel.

A. Edge detection: A standard implementation of Canny edge detector with hysteresis [8] is used to find the edge pixels of the considered bone structure from the input images. The detected edge pixels are further processed using the knowledge about the X-ray machine. Detected edge pixel whose intensity is below some threshold or whose position is of a certain distance away from the image center is eliminated.

B. Apparent contour extraction: For fast extraction a smooth apparent contour from a given surface model, we use the approach introduced by Hertzmann and Zorin [9]. This approach first constructs a dual representation of the given surface mesh in a four-dimensional (4D) space based on the position and tangent planes of every vertex. The focal point's dual (a plane in 4D) intersects with the mesh triangles dual. Before hand, the approach normalizes the dual vertices using the $l_{\infty}$ norm so that the vertices end up on one of the unit hyper-cube's sides. This reduces the problem to intersecting the triangles on a hypercube's sides with the focal point's dual plane, whose solution can be accelerated greatly using octree data structure. An example is given in Fig. 1.

Then, for a point $\Omega_{j}^{s},(j=0,1, \ldots, M-1)$ on the extracted apparent contours using the projection parameters of the $s$ th input X-ray image, we do a forward projection of this point onto image $s$ to get its 2D position $A_{j}^{s}$. Each 2D point in $\left\{A_{j}^{s}\right\}$ is thus associated to a $3 \mathrm{D}$ point in $\left\{\Omega_{j}^{s}\right\}$. Next, we will describe an iterative matching process for build $2 \mathrm{D}$ association between the points in $\left\{A_{j}^{s}\right\}$ and the detected edge pixels in the X-ray image $s$.

C. Iterative non-rigid $2 D$ matching process: Following the general framework of point matching proposed in [10], we also formulate the 2D matching as a two-stage process: correspondence and spatial mapping. TPS is used here for parameterization of the spatial mapping. But unlike [10, we solve the correspondence problem differently. To make the description simple, we denote 


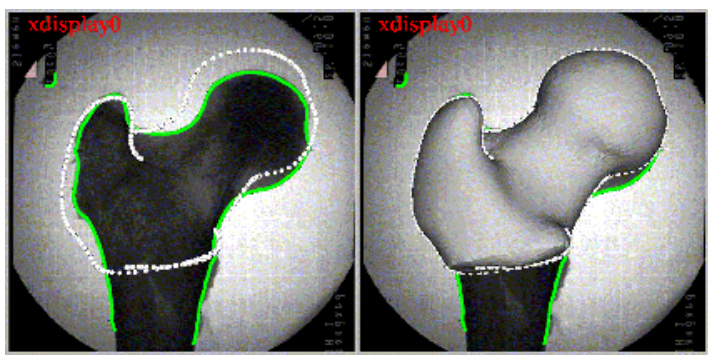

Fig. 1. Example of extracting apparent contours (white) and edge pixels (green)

the detected edge pixels in image $s$ as $I^{s}=\left\{I_{i}^{s}, i=0,1, \ldots, Q-1\right\}$ and the 2D projection of the apparent contours as $A^{s}=\left\{A_{j}^{s}, j=0,1, \ldots, M-1\right\}$. Here we focus on 2D matching in one image. The overall correspondence is established by combining matched point pairs found in all input images.

Definition 1: Injective Nearest-neighbor. A point $I_{i}^{s}$ can only be matched to at most one point of $A^{s}$ and this point must be the closest one in $A^{s}$ to $I_{i}^{s}$.

Definition 2: Cross-matching. Assume there are two matched pairs $\left(I_{i}^{s}, A_{j}^{s}\right)$ and $\left(I_{m}^{s}, A_{n}^{s}\right)$. If the line segmentation from $I_{i}^{s}$ to $A_{j}^{s}$ intersects at a point with the line segment from $I_{m}^{s}$ to $A_{n}^{s}$, we define this event as cross-matching.

Definition 3: Symmetric Injective Nearest-neighbor (SIN). $I_{i}^{s}$ and $A_{j}^{s}$ is a symmetric injective nearest-neighbor if and only if $A_{j}^{s}$ is the closest point in $A^{s}$ to $I_{i}^{s}$ and $I_{i}^{s}$ is the closest point in $I^{s}$ to $A_{j}^{s}$.

Definition 4: Symmetric Injective Nearest-neighbor Mapping Operator (SIN-MO). We define the action of finding a number of SIN's from two points set as symmetric inject nearest-neighbor mapping operator.

Claim 1: If we apply SIN-MO on two point sets to find a number of matched point pairs, all of them are one-to-one mapping (proof omitted).

Claim 2: If we apply SIN-MO on two point sets to find a number of matched point pairs, there is no cross-matching (proof omitted).

The iterative non-rigid 2D matching process can be described as follows.

Input: two point sets $I^{s}$ and $A^{s}$, and a weight parameter $\lambda$.

Output: a list of $2 \mathrm{D}$ matched point pairs.

Initialization: We first calculate the centroids of $I^{s}$ and $A^{s}$, and then translate $A^{s}$ so that its centroid is aligned with the centroid of $I^{s}$.

Iteration: It is a dual update process taking points in $I^{s}$ as references.

Stage 1: Update the correspondence. Apply SIN-MO on $I^{s}$ and $A^{s}$ to find a number of SINs and denote the set of SINs as $\left\{\left(I_{a}^{s}, A_{a}^{s}\right) ; a=0,1, \ldots, K-1\right\}$.

Stage 2: Update the positions of all points in $A^{s}$. This is done in a two-step procedure.

Step 2.1. Compute a 2D TPS-based spatial mapping $f$ using the estimated set of SINs by minimizing the following cost function: 


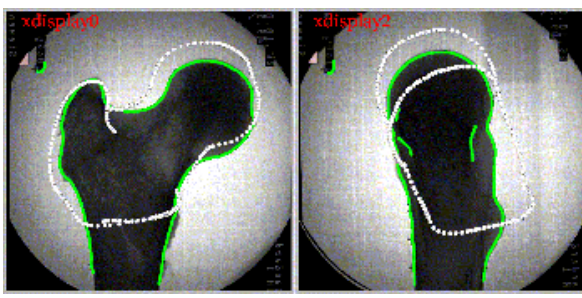

(a) Before building correspondence

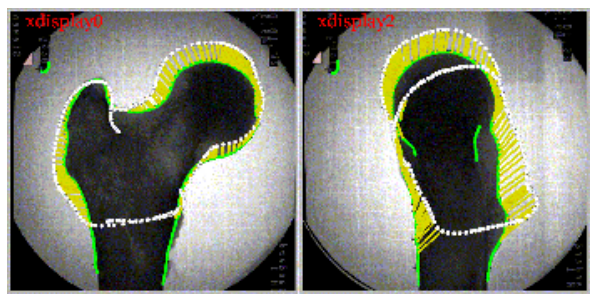

(b) After building correspondences

Fig. 2. Establishing 2D/3D correspondence; detected edge pixels (green), extracted apparent contours (white), and the estabilished correspondences (linked with yellow line segment for visualization purpose)

$$
\sum_{a=0}^{K-1}\left\|I_{a}^{s}-f\left(A_{a}^{s}\right)\right\|^{2}+\lambda \iint\left[\left(\frac{\partial^{2} f}{\partial x^{2}}\right)^{2}+\left(\frac{\partial^{2} f}{\partial x \partial y}\right)^{2}+\left(\frac{\partial^{2} f}{\partial x^{2}}\right)^{2}\right] d x d y
$$

Step 2.2. Update the positions of all points in $A^{s}$ based on the estimated TPS transformation $f$.

Repeat stage 1 and 2 a certain times (e.g. 30) or until convergence.

D. 3D point pair building: Assume that we have found a set of $2 \mathrm{D}$ matched point pairs $\left\{\left(I_{b}^{s}, A_{b}^{s}\right) ; b=0,1, \ldots, L-1\right\}$, we are trying to build the corresponding $3 \mathrm{D}$ point pairs as follows. For a $2 \mathrm{D}$ point $I_{b}^{s}$, we can find a projection ray $r_{b}^{s}$ emitting from the focal point of image $s$ through point $I_{b}^{s}$. Additionally, for its matched point $A_{b}^{s}$, we always know its associated $3 \mathrm{D}$ point $\Omega_{b}^{s}$ on the apparent contour of the model whose projection onto the image s is $A_{b}^{s}$. By computing a point $v_{b}^{s}$ on the ray $r_{b}^{s}$ that has the shortest distance to $\Omega_{b}^{s}$, we can build a $3 \mathrm{D}$ point pair $\left(v_{b}^{s}, \Omega_{b}^{s}\right)$. Combining all these $3 \mathrm{D}$ point pairs, we can establish $2 \mathrm{D} / 3 \mathrm{D}$ correspondence between the input images and a 3D model estimated from the PDM. An example is given in Fig. 2.

\section{3D/3D Reconstruction}

Using the developed algorithm for establishing correspondence, we can always find a set of $3 \mathrm{D}$ point pairs given an initial model state, i.e., the initial scale and the initial pose parameters of our point distribution model. The problem of surface reconstruction is then solved optimally in three stages as presented in our previous work [4]: affine registration, statistical extrapolation, and regularized shape deformation.

A. Affine registration: This is the only stage that is solved by iteration. In this stage, the scale and the rigid registration transformation between the mean model of the PDM and the input images are iteratively determined using a generalization of the Iterative Closest Point (ICP) algorithm developed by Guéziec et al. 11. The difference between this algorithm and the traditional ICP algorithm is that in each iteration we need to set up a set of new point pairs using the present algorithm for building $2 \mathrm{D} / 3 \mathrm{D}$ correspondences. 
B. Statistical extrapolation: Based on the estimated scale and pose information from the first stage, we can use the correspondence establishing algorithm to obtain a set of $n$ 3D point pairs from all input images. Let's denote the points computed from the image data as $\mathrm{v}^{\prime}=\left\{v_{i}^{\prime}=\left(x_{i}^{\prime}, y_{i}^{\prime}, z_{i}^{\prime}\right) ; i=0,1, \ldots, n-1\right\}$ and call them the image points. Let's further denote those points on the mean model of the PDM as $\overline{\mathrm{x}}^{\prime}=\left\{\left(\overline{\mathrm{x}}_{j}\right)_{i} ; 0 \leq j \leq N-1 ; i=0,1, \ldots, n-1\right\}$ and call them the model points, where $N$ is the number of points of the mean model; $n$ is the number of the matched point pairs. $\left(\overline{\mathrm{x}}_{j}\right)_{i}$ means that the $j$ th model point $\overline{\mathrm{x}}_{j}$ on the mean model $\overline{\mathrm{x}}$ is the closest point to the $i$ th image point $v_{i}^{\prime}$. The statistical extrapolation is formulated as the minimization of the following joint cost function:

$$
\begin{aligned}
& E_{\alpha}\left(\overline{\mathrm{x}}^{\prime}, \mathrm{v}^{\prime}, \mathrm{x}\right)=\rho \cdot \log (3 n) \cdot E\left(\overline{\mathrm{x}}^{\prime}, \mathrm{v}^{\prime}, \mathrm{x}\right)+\mathrm{E}(\mathrm{x}) ; \mathrm{x}=\overline{\mathrm{x}}+\sum_{k=0}^{m-2} \alpha_{k} \cdot \mathbf{p}_{k} \\
& E\left(\overline{\mathrm{x}}^{\prime}, \mathrm{v}^{\prime}, \mathrm{x}\right)=\left(n^{-1}\right) \cdot \sum_{i=0}^{n-1}\left\|v_{i}^{\prime}-\left(\left(\overline{\mathrm{x}}_{j}\right)_{i}+\sum_{k=0}^{m-2} \alpha_{k} \cdot \mathbf{p}_{k}(j)\right)\right\|^{2} \\
& E(\mathrm{x})=(1 / 2) \cdot \sum_{k=0}^{m-2}\left(\alpha_{k}^{2} / \sigma_{k}^{2}\right)
\end{aligned}
$$

where the first term is the likelihood energy term and the second term is the prior energy term (or the stabilization term), used to constrain the estimated shape to a realistic result. $\rho$ is a parameter that controls the relative weighting between these two terms. $\mathbf{p}_{k}(j)$ is the $j$ th tuple of the kth shape basis eigenvector.

For details about how to solve Eq. (6), we refer to our previous work 4 .

C. Regularized shape deformation: Similar to the second stage, we first set up image-to-model correspondence between the input images and a template surface model estimated through optimizing Eq. (6). To keep the same notation, let's assume that the image points are $\mathrm{v}^{\prime}=\left\{v_{i}^{\prime}=\left(x_{i}^{\prime}, y_{i}^{\prime}, z_{i}^{\prime}\right) ; i=0,1, \ldots, l-1\right\}$ and that the model points are $\mathrm{v}=\left\{v_{i}=\left(\mathrm{x}_{j}\right)_{i}=\left(x_{i}, y_{i}, z_{i}\right) ; i=0,1, \ldots, l-1\right\}$, where $l$ is the number of point pairs and $\left(\mathrm{x}_{j}\right)_{i}$ means that the $j$ th model point $\mathrm{x}_{j}$ on the statistically extrapolated surface $\mathrm{x}$ is the closest point to the $i$ th image point $v_{i}^{\prime}$. The regularized shape deformation is described as a regression problem of finding a spatial transform $\mathrm{t}: \Re^{3} \rightarrow \Re^{3}$ that minimizes following cost function:

$$
E(\mathrm{t})=\left(l^{-1}\right) \cdot \sum_{i=0}^{l-1}\left\|v_{i}^{\prime}-\mathrm{t}\left(v_{i}\right)\right\|^{2}+\tau \cdot \frac{\log (m)}{\log (3 l)} \cdot L[\mathrm{t}]
$$

where $\tau \geq 0$ is a parameter controlling the fitting quality and the regularization constraint; $m$ is the number of training surfaces; $\mathrm{t}(\mathrm{v})=\left\{\mathrm{t}\left(v_{i}\right)\right\}$ are the results of applying the mapping on the model points and $L[\mathrm{t}]$ is a thin-plate splines based regularization functional defined on the nonlinear mapping $t$ and has following form [12]:

$$
\begin{aligned}
& L[\mathrm{t}]=\iiint_{\Re^{3}}(B(\mathrm{t})) d x d y d z \\
& B(\cdot)=\left(\frac{\partial^{2}}{\partial x^{2}}\right)^{2}+\left(\frac{\partial^{2}}{\partial x \partial y}\right)^{2}+\left(\frac{\partial^{2}}{\partial y^{2}}\right)^{2}+\left(\frac{\partial^{2}}{\partial y \partial z}\right)^{2}+\left(\frac{\partial^{2}}{\partial z^{2}}\right)^{2}+\left(\frac{\partial^{2}}{\partial z \partial x}\right)^{2}
\end{aligned}
$$

For details about how to solve Eq. (7), we refer to our previous work [4. 


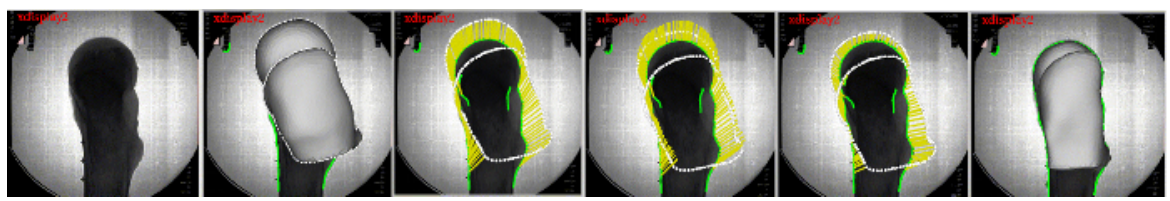

Fig. 3. Different stages of reconstruction. First: one of the acquired images. Second: the initialization of the mean model of the PDM. Third: after establishing image-to-model correspondence. Forth: after 3D paired point matching. Fifth: after re-establishing correspondence; Sixth: the final reconstruction result after a series of computations

Table 1. Reconstruction errors when different number of images were used

\begin{tabular}{|c|c|c|c|c|c|c|c|c|c|c|c|}
\hline \multicolumn{12}{|c|}{ Reconstruction errors when only AP and LM images were used for each bone } \\
\hline Bone Index & No. 1 & No. 2 & No. 3 & No. 4 & No. 5 & $\overline{\text { No. } 6}$ & No. 7 & No. 8 & No. 9 & No. 10 & No. 11 \\
\hline Median (mm) & 1.3 & 0.8 & 1.5 & 1.0 & 1.3 & 1.0 & 1.1 & 1.0 & 0.8 & 1.1 & 1.2 \\
\hline Mean (mm) & 1.5 & 0.8 & 1.4 & 1.3 & 1.4 & 1.2 & 1.2 & 1.2 & 1.0 & 1.1 & 1.6 \\
\hline \multicolumn{12}{|c|}{ Reconstruction errors when all three images were used for each bone } \\
\hline Bone Index & No. 1 & No. 2 & No. 3 & No. 4 & No. 5 & No. 6 & No. 7 & No. 8 & No. 9 & No. 10 & No. \\
\hline Median (mm) & 1.3 & 0.7 & 0.7 & 1.1 & 1.0 & 1.1 & 0.8 & 0.9 & 0.7 & 1.0 & 0.9 \\
\hline Tean $(\mathrm{mm})$ & 1.3 & 0.7 & $\overline{0.8}$ & $\overline{1.2}$ & 1.1 & 1.1 & 1.1 & $\overline{0.9}$ & $\overline{0.9}$ & 1.1 & $\overline{1.2}$ \\
\hline
\end{tabular}

\section{$5 \quad$ Experiments and Results}

We designed and conducted experiments on 11 cadaveric femurs (Note: none of them has been included for constructing the PDM) with different shape to validate the present reconstruction scheme. Three fluoroscopic images were acquired for each bone: the first one along the anterior-posterior (AP) direction, the second one along the lateral-medial (LM) direction, and the last one from an oblique angle in between the AP and the LM directions. All acquired images were calibrated and registered to the associated reference coordinate system. And in all reconstructions, three controlling parameters are set as follows: $\lambda=0.5, \rho=0.05$, and $\tau=0.05$.

Two experiments using different number of images were performed for each bone. In the first experiment only AP and LM images were used to reconstruct the surface of the bone. In the second one, all three images were used.

The reconstruction accuracies were evaluated by randomly digitizing 100 200 points from each surface of the cadaveric specimen and then computing the distance from those digitized points to the associated surface reconstructed from the images. The median and mean reconstruction errors of both experiments are presented in Table 1. An average mean reconstruction error of $1.2 \mathrm{~mm}$ was found when only AP and LM images were used for each bone. It decreased to $1.0 \mathrm{~mm}$ when three images were used. Different stages of one reconstruction example are presented in Fig. 3. 


\section{Conclusions}

In this paper, we have presented a $2 \mathrm{D} / 3 \mathrm{D}$ reconstruction scheme combining statistical extrapolation and regularized shape deformation with an iterative imageto-model correspondence establishing algorithm, and showed its application to reconstruct the surface of proximal femur. The advantages of the present imageto-model correspondence establishing algorithm include the robustness with respect to certain outliers and automatic exclusion of cross matching, which is an important property for preservation of topology. Experiments performed on 11 cadaveric femurs demonstrated the accuracies of the present scheme. It holds the potential to increase reconstruction accuracy when more images are used. Our future plan is to improve the algorithm for image feature detection with the final goal of applying the present reconstruction scheme to real situation.

\section{References}

1. Fleute M., Lavallée S.: Nonrigid 3D/2D registration of images using a statistical model. Lecture Notes in Computer Science, Springer 1679 (1999) 138-147

2. Benameur S., Mignotte M., Parent S. et al.: 3D/2D registration and segmentation of scoliotic vertebra using statistical models. Comput Med Imag Grap 27 (2003) 321-337

3. Benameur S., Mignotte M., Parent S., et al.: A hierarchical statistical modeling approach for the unsupervised 3D reconstruction of the scoliotic spine. In ICIP'04 (2004) 561-563

4. Zheng G., Rajamani K.T. and Nolte L.-P.: Use of a dense surface point distribution model in a three-stage anatomical shape reconstruction from sparse information for computer-assisted orthopaedic surgery: a preliminary study. In ACCV'06, Lecture Notes in Computer Science, Springer 3852 (2006) 52-60

5. Styner M., Rajamani K.T., Nolte L.-P., Zsemlye G., Szkely G., Taylor C.J., Davis R.H.: Evaluation of 3D correspondence methods for modeling building. In IPMI'03, Lecture Notes in Computer Science, Springer 2732 (2003) 63-75

6. Brechbuehler C., Gerig G., Kuebler O.: Parameterization of Closed Surfaces for 3D Shape Description. Comput Vision and Image Under 61 (1995) 154-170

7. Davies R. H., Twining C. H., et al.: 3D statistical shape models using direct optimization of description length. In ECCV'02, Lecture Notes in Computer Science, Springer 2352 (2002) 3-20

8. Canny J.: A computational approach to edge detection. IEEE T Pattern Anal 8 (1986) 679-698

9. Hertzmann A., Zorin D.: Illustrating smooth surfaces. In SIGGRAPH'00, (2000), $517-526$

10. Chui H., Rambo J., Duncan J., Schultz R., and Rangarajan A.: Registration of cortical anatomical structures via robust 3D point matching. In: IPMI'99, Lecture Notes in Computer Science, Springer 1613 (1999), 168-181

11. Guéziec A., Kazanzides P., Williamson B., and Taylor R.H.: Anatomy-based registration of CT-scan and intraoperative X-ray images for guiding a surgical robot. IEEE T Med Imaging, 17 (1998), 715-728

12. Bookstein F.: Principal warps: thin-plate splines and the decomposition of deformations. IEEE T Pattern Anal, 11 (1989), 567-585 